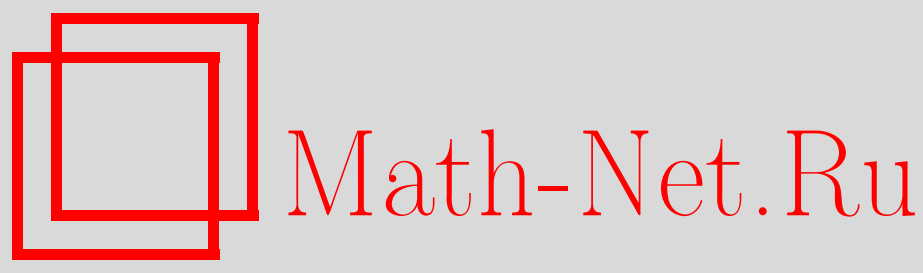

А. В. Киселев, Й. В. ван де Лёр, Вариационные алгеброиды Ли и гомологические эволюционные векторные поля, ТМФ, 2011, том 167, номер 3, 432-447

DOI: https://doi.org/10.4213/tmf6652

Использование Общероссийского математического портала Math-Net.Ru подразумевает, что вы прочитали и согласны с пользовательским соглашением http: //www . mathnet.ru/rus/agreement

Параметры загрузки:

IP : 54.174 .149 .18

26 апреля 2023 г., 15:39:46

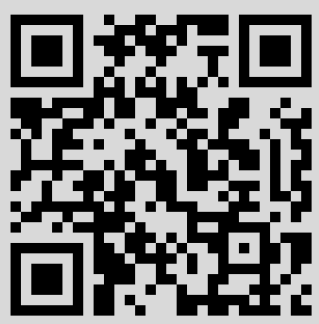




\title{
ВАРИАЦИОННЫЕ АЛГЕБРОИДЫ ЛИ И ГОМОЛОГИЧЕСКИЕ ЭВОЛЮЦИОННЫЕ ВЕКТОРНЫЕ ПОЛЯ
}

\begin{abstract}
Дано определение алгеброидов Ли над пространствами бесконечных струй и получена его эквивалентная формулировка в терминах гомологических эволюционных векторных полей.
\end{abstract}

Ключевые слова: алгеброид Ли, БРСТ-дифференциал, пуассонова структура, интегрируемые системы, теория струн.

\section{1. ВВЕДЕНИЕ}

Понятие алгеброидов Ли над гладкими многообразиями является важным в дифференциальной геометрии (в особенности, в пуассоновой геометрии) и возникает в различных моделях математической физики (например, в пуассоновых сигма-моделях). Мы обобщаем классическое определение алгеброидов Ли над гладкими многообразиями [1] и вводим конструкцию вариационных алгеброидов Ли над пространствами бесконечных струй. Эти структуры мы определяем как стандартным образом, через векторные расслоения, так и в терминах гомологических векторных полей $Q^{2}=0$ на суперрасслоениях бесконечных струй, доказывая затем эквивалентность двух определений. Предложенное нами обобщение классической конструкции в явной форме учитывает ту геометрию, которая возникает при отображениях гладких многообразий. Таким образом, развиваемый в настоящей работе вариационный подход более полно отражает геометрию струн в пространстве-времени [2].

Сначала мы напоминаем (весьма кратко) определение обычных алгеброидов Ли; все дальнейшие подробности см. в работе [1] (а также в обзорах [3] и приведенных там ссылках). Общеизвестными примерами алгеброидов Ли над обычными многообразиями служат касательное расслоение или структура пуассонова алгеброида на кокасательном расслоении к пуассонову многообразию. Вместе с тем собственно алгебры Ли являются тривиальными примерами алгеброидов Ли над точкой.

${ }^{*}$ Mathematical Institute, University of Utrecht, Utrecht, The Netherlands.

E-mail: A.V.Kiselev@rug.nl, J.W.vandeLeur@uu.nl

$\dagger$ Johann Bernoulli Institute for Mathematics and Computer Science, University of Groningen, Groningen, The Netherlands. 
По аналогии мы будем трактовать гладкие многообразия $M^{m}$ как (крайне бедные) "пространства бесконечных струй" отображений точки в эти многообразия, которые становятся "слоями" в "расслоениях" $\pi: M^{m} \rightarrow\{\mathrm{pt}\}$. Такое донельзя нестрогое (но в равной степени продуктивное!) описание служит отправной точкой наших рассуждений. Ниже мы установим, во что превращаются алгеброиды Ли над $M^{m}$ при переходе от точки в качестве базы расслоения к $n$-мерному многообразию $\Sigma^{n}$, которое задает настоящее расслоение струй $J^{\infty}\left(\Sigma^{n} \rightarrow M^{m}\right)$.

Результаты настоящей статьи изложены в следующем порядке. Прежде всего, мы отмечаем, что буквальный перенос классического определения для гладких многообразий на случай расслоений бесконечных струй невозможен, поскольку над ними с самого начала теряет силу правило Лейбница (точно так же, как для коммутаторов эволюционных векторных полей или для вариационной скобки Пуассона [4]). По этой причине в разделе 2 мы принимаем за определение должным образом выбранное следствие классического определения, а именно замкнутость образов якорей относительно коммутирования (примечательно, что это свойство часто для удобства формулируют как одну из составляющих определения алгеброида Ли вместо того, чтобы его вывести, см., например, работы [1], [5] в противоположность [6], [7]). На этом языке переформулировка основного результата нашей работы [8] звучит так: непериодические двумеризованные уравнения Тоды, ассоциированные с полупростыми комплексными алгебрами Ли [9], - представители обширного класса гиперболических лагранжевых систем лиувиллева типа [10] - суть вариационные алгеброиды Ли.

ЗАмЕчАниЕ 1. Необходимо уточнить, что определение алгеброидов Ли над пространствами конечных струй сечений касательного расслоения $\pi: T \Sigma \rightarrow \Sigma^{n}$ было дано в работе [11]. В настоящей статье, напротив, векторное расслоение $\pi$ может быть каким угодно. Не ограниченные одним лишь касательным расслоением (как в [11]), рассматриваемые ниже модельные примеры имеют совершенно иную природу. А именно, мы заимствуем их из геометрии дифференциальных уравнений, рассматривая, в частности, гамильтоновы (не)эволюционные системы [12] или двумеризованные цепочки Тоды [9], решения которых - это $r$ функций двух переменных, a $r$ - ранг произвольной полупростой комплексной алгебры Ли. Иными словами, мы изучаем общий случай, когда размерности базы и слоя расслоения $\pi$, вообще говоря, никак не связаны между собой.

В разделе 3 мы строим представление структуры вариационного алгеброида Ли $\mathfrak{A}$, определенного описанным выше способом, в терминах гомологического эволюционного векторного поля $Q$ на расслоении бесконечных струй сечений суперрасслоения ПА, понимаемого как векторное расслоение с противоположной четностью слоев. Данный переход также нетривиален, поскольку правило Лейбница, используемое при доказательстве эквивалентности двух картин в случае классического определения, теперь утрачено. Здесь же мы налагаем естественное условие невырожденности на вариационные якори, чтобы нечетное поле $Q$ было корректно определено. Для иллюстрации мы выбираем в качестве вариационных якорей гамильтоновы дифференциальные операторы; так мы получаем поля $Q$, которые в свою очередь сами оказываются гамильтоновыми относительно соответствующих 
им вариационных пуассоновых бивекторов и канонической симплектической структуры, рассмотренной в работе [13].

На протяжении всей статьи основным полем служит поле $\mathbb{R}$ вещественных чисел, и мы предполагаем, что все отображения бесконечно гладкие. Мы также будем считать, что все дифференциальные операторы в полных производных локальны, т. е. полиномиальны по полным производным. Кроме того, предположим, что все пространства чисто четные: ни одно многообразие не является супермногообразием, кроме тех, про которые сказано обратное, - тех, которые возникают за счет явной смены четности с помощью преобразования П. Мы применяем стандартные конструкции из геометрии дифференциальных уравнений [4], [14]; для удобства мы напоминаем требуемые понятия в начале раздела 2. Поскольку все рассуждения проводятся локально, мы с самого начала переходим к соответствующим картам атласа и вместо расслоений струй отображений $\Sigma^{n} \rightarrow M^{m}$ многообразий рассматриваем пространства струй сечений $m$-мерных векторных расслоений $\pi: E^{m+n} \rightarrow \Sigma^{n}$. По существу, мы даем все определения для одних лишь пространств бесконечных струй, не делая также различия между автономными эволюционными уравнениями и эволюционными векторными полями (что общепринято). Выяснение всех деталей тонкого различия между определениями рассматриваемых здесь структур на пустых пространствах струй и на дифференциальных уравнениях потребовало бы значительно более объемного изложения; этот вопрос будет рассмотрен в другой статье. Пока что мы отсылаем читателя к обзору [12].

Наши обозначения следуют статьям [8], [15], [16] и в целом согласуются с обозначениями из работ [3], [4], [14]. Настоящая работа является продолжением публикаций [8], [16], в которых приведены мотивирующие примеры вариационных алгеброидов Ли. Некоторые из результатов, рассматриваемых здесь по возможности досконально, были кратко анонсированы ранее в препринте [17].

Обычные алгеброиды Ли: обзор. Пусть $M^{m}$ - гладкое вещественное $m$-мерное многообразие $(1 \leqslant m \leqslant+\infty)$ и $T M \rightarrow M^{m}$ - его касательное расслоение.

ОПРЕДЕЛЕНИЕ 1 [1]. Алгеброидом Ли над многообразием $M^{m}$ называется векторное расслоение $\xi: \Omega^{d+m} \rightarrow M^{m}$, пространство сечений которого, обозначаемое через $\Gamma \Omega$, наделено структурой $[,]_{A}$ алгебры Ли и морфизмом расслоений $A: \Omega \rightarrow$ $T M$, называемым якорем, так, что выполнено правило Лейбница

$$
[f \cdot \mathcal{X}, \mathcal{Y}]_{A}=f \cdot[\mathcal{X}, \mathcal{Y}]_{A}-(A(\mathcal{Y}) f) \cdot \mathcal{X}
$$

для любых $\mathcal{X}, \mathcal{Y} \in \Gamma \Omega$ и произвольной функции $f \in C^{\infty}\left(M^{m}\right)$.

По существу, якорь в алгеброиде Ли является подчиненным некоторым дополнительным условиям послойно линейным отображением из наперед заданного векторного расслоения над гладким многообразием $M^{m}$ в его касательное расслоение.

Лемма 1 [6]. Якорь А отображает скобку $[,]_{\text {А }}$ сечений векторного расслоения $\xi$ в скобку Ли [, ] сечений касательного расслоения над многообразием $M^{m}$.

Это свойство является следствием правила Лейбница (1) и тождества Якоби для структуры алгебры Ли $[,]_{A}$ на $\Gamma \Omega$.

ЛЕмма 2 [1]. Структуру алгеброида Ли на пространстве $\Omega$ можно эквивалентным образом задать как гомологическое векторное поле $Q$ на П $\Omega$ (чтобъ 
получить новое суперрасслоение $\Pi \Omega$ над $M^{m}$, нужно изменить четность слоев расслоения $\Omega$ на противоположную). Гомологическое векторное поле, являющееся дифберенциалом на пространстве $C^{\infty}(\Pi \Omega)=\Gamma\left(\bigwedge^{\bullet} \Omega^{*}\right)$, равно

$$
Q=A_{i}^{\alpha}(u) b^{i} \frac{\partial}{\partial u^{\alpha}}-\frac{1}{2} b^{i} c_{i j}^{k}(u) b^{j} \frac{\partial}{\partial b^{k}}, \quad[Q, Q]=0 \quad \Leftrightarrow \quad 2 Q^{2}=0
$$

¿əe:

- $\left(u^{\alpha}\right)$ - система локальных координат в окрестности точки $u \in M^{m}$;

- $\left(p^{i}\right)$ - локальные координаты вдоль $d$-мерных слоев в $\Omega$, a $\left(b^{i}\right)$ - соответствующие им координаты на $\Pi \Omega$;

- формула $\left[e_{i}, e_{j}\right]_{A}=c_{i j}^{k}(u) e_{k}$ задает структурные константы в $d$-элементном базисе локальных сечений расслоения Г $\Omega$ над точкой $u$, a $A\left(e_{i}\right)=A_{i}^{\alpha}(u) \cdot \partial / \partial u^{\alpha}-$ образ вектора е

НАБРосоК докАЗАтЕЛьСтВА. Антикоммутатор $[Q, Q]=2 Q^{2}$ нечетного векторного поля $Q$ с самим собой - это также векторное поле. В нем коэффициент при $\partial / \partial u^{\alpha}$ равен нулю благодаря тому, что (в силу леммы 1) $A$ является гомоморфизмом алгебр Ли. Равенство нулю коэффициента при $\partial / \partial b^{q}$ устанавливается за три шага. Заметим, во-первых, что действие второго слагаемого в (2) само на себя (причем для векторных полей справедливо градуированное правило Лейбница) приводит к численному множителю $1 / 4$, но он удвоится вследствие кососимметричности структурных констант $c_{i j}^{k}$. Во-вторых, распозна́ем правую часть правила Лейбница (1) с величинами $c_{i j}^{k}(u)$, подставленными в качестве функции $f \in C^{\infty}\left(M^{m}\right)$, в слагаемом

$$
\frac{1}{2} \sum_{i, j, n} b^{i} b^{j} b^{n}\left(-A_{n}^{\alpha} \frac{\partial}{\partial u^{\alpha}}\left(c_{i j}^{q}(u)\right)+c_{i j}^{\ell} c_{\ell n}^{q}\right) \cdot \frac{\partial}{\partial b^{q}} .
$$

В-третьих, заметим, что ииклическая перестановка $i \rightarrow j \rightarrow n \rightarrow i$ нечетных переменных $b^{i}, b^{j}, b^{n}$ не приводит к смене знака в выражении (3). Следовательно, утроим его, беря сумму по циклическим перестановкам (но и одновременно разделим на три), и в качестве коэффициента при $\partial / \partial b^{q}$ получим тождество Якоби для скобки $[,]_{A}$ :

$$
\frac{1}{2} \cdot \frac{1}{3} \cdot \sum_{\circlearrowright}\left[\left[e_{i}, e_{j}\right]_{A}, e_{n}\right]_{A}=0 .
$$

Стоящий в правой части ноль дает искомый коэффициент. Итак, $Q^{2}=0$.

\section{2. ВАРИАЦИОННЫЕ АЛГЕБРОИДЫ ЛИ}

Для полноты изложения напомним обозначения. Пусть $\Sigma^{n}-n$-мерное ориентируемое гладкое вещественное многообразие; рассмотрим над ним векторное расслоение $\pi: E^{m+n} \underset{M^{m}}{\rightarrow} \Sigma^{n}$ с $m$-мерными слоями $M^{m} \ni u=\left(u^{1}, \ldots, u^{m}\right)$. Пространство бесконечных струй сечений расслоения $\pi$ обозначим через $J^{\infty}(\pi)$ и положим $\pi_{\infty}: J^{\infty}(\pi) \rightarrow \Sigma^{n}$. Соответствующие координаты вдоль слоев суть $u_{\sigma},|\sigma| \geqslant 0$. Введем обозначение $[u]$, выражающее дифференциальную зависимость от переменных $u$ и их производных не выше некоторого произвольно высокого, но конечного порядка; положим $\mathcal{F}(\pi):=C^{\infty}\left(J^{\infty}(\pi)\right)$, понимая эту алгебру сообразно сделанной выше оговорке (как индуктивный предел фильтрованных алгебр, см. [4]). 
Пусть $\xi: N \rightarrow \Sigma^{n}$ - еще одно векторное расслоение над той же базой $\Sigma^{n}$; используя $\pi_{\infty}$, построим из него индуцированное расслоение $\pi_{\infty}^{*}(\xi): N \times_{\Sigma^{n}} J^{\infty}(\pi) \rightarrow J^{\infty}(\pi)$. По определению $\mathcal{F}(\pi)$-модуль сечений $\Gamma\left(\pi_{\infty}^{*}(\xi)\right)=\Gamma(\xi) \otimes_{C^{\infty}\left(\Sigma^{n}\right)} \mathcal{F}(\pi)$ называется горизонтальным [18].

ПримеР 1. Со всяким пространством струй $J^{\infty}(\pi)$ канонически ассоциированы три горизонтальных $\mathcal{F}(\pi)$-модуля (см., например, [18]). Прежде всего, положим $\xi:=\pi$ и получим $\mathcal{F}(\pi)$-модуль $\Gamma\left(\pi_{\infty}^{*}(\pi)\right)=\Gamma(\pi) \otimes_{C^{\infty}\left(\Sigma^{n}\right)} \mathcal{F}(\pi)$. Для краткости обозначим его через $\varkappa(\pi) \equiv \Gamma\left(\pi_{\infty}^{*}(\pi)\right)$. Принадлежащие ему сечения $\varphi \in \varkappa(\pi)$ находятся во взаимно однозначном соответствии с $\pi$-вертикальными эволюционными дифференцированиями $\partial_{\varphi}=\sum_{\sigma} d^{|\sigma|} \varphi / d x^{\sigma} \cdot \partial / \partial u_{\sigma}$ на $J^{\infty}(\pi)$, где

$$
\frac{d}{d x}=\frac{\partial}{\partial x}+u_{x} \frac{\partial}{\partial u}+\cdots
$$

- полная производная. Для всех $\psi$ таких, что выражение $\partial_{\varphi}(\psi)$ определено, их линеаризации $\ell_{\psi}^{(u)}$ заданы формулой $\ell_{\psi}^{(u)}(\varphi)=\partial_{\varphi}(\psi)$, где $\varphi \in \varkappa(\pi)$.

Далее, пусть $\xi-q$-я внешняя степень кокасательного расслоения $T^{*} \Sigma$ при $q \leqslant n$. Рассмотрим индуцированное расслоение $\pi_{\infty}^{*}(\xi)$ и получим горизонтальный $\mathcal{F}(\pi)$-модуль $\bar{\Lambda}^{q}(\pi)$ его сечений, которые называются горизонтальными q-формами на пространстве струй $J^{\infty}(\pi)$. В локальных координатах эти сечения можно записать в виде $h(x,[u]) \cdot d x^{i_{1}} \wedge \cdots \wedge d x^{i_{q}}$, где $h \in \mathcal{F}(\pi)$ и $1 \leqslant i_{1}<\cdots<i_{q} \leqslant n$.

Наконец, рассмотрим модуль $\bar{\Lambda}^{n}(\pi) \pi$-горизонтальных форм высшей степени на $J^{\infty}(\pi)$. Обозначим через $\hat{\varkappa}(\pi)=\operatorname{Hom}_{\mathcal{F}(\pi)}\left(\varkappa(\pi), \bar{\Lambda}^{n}(\pi)\right)$ горизонтальный $\mathcal{F}(\pi)$-модуль, двойственный к $\varkappa(\pi)$.

ЗАмечАниЕ 2. Структура слоев расслоения $\xi$ может быть сложной, а их размерность - бесконечной. Приведенный ниже пример является в определенном смысле каноническим и формализует геометрию двумеризованных систем Тоды [8]. А именно, пусть $\zeta: I^{r+n} \underset{W^{r}}{\rightarrow} \Sigma^{n}$ - векторное расслоение с $r$-мерными слоями, на которых $w=\left(w^{1}, \ldots, w^{r}\right)-$ локальные координаты. Рассмотрим расслоение бесконечных струй $\xi_{\infty}: J^{\infty}(\xi) \rightarrow \Sigma^{n}$ и положим по определению либо $\xi=\zeta_{\infty} \circ \zeta_{\infty}^{*}(\zeta): \varkappa(\xi) \rightarrow \Sigma^{n}$, либо $\xi=\zeta_{\infty} \circ \zeta_{\infty}^{*}(\widehat{\zeta}): \hat{\varkappa}(\xi) \rightarrow \Sigma^{n}$. Чтобы избежать нагромождения формул, всюду далее мы будем использовать сокращенное обозначение $\Gamma \Omega\left(\xi_{\pi}\right)$ для рассматриваемого $\mathcal{F}(\pi)$-модуля.

По определению отображения между $\mathcal{F}(\pi)$-модулями называются дифференииальными операторами в полных производных, если они являются суммами композиций $\mathcal{F}(\pi)$-линейных отображений и поднятий векторных полей с базы $\Sigma^{n}$ на $J^{\infty}(\pi)$ при помощи связности Картана $\partial / \partial x \mapsto d / d x$. Основные объекты нашего исследования - матричные линейные дифференциальные операторы в полных производных, принимающие значения в алгебре Ли $\mathfrak{g}(\pi)=(\varkappa(\pi),[]$,$) .$

Пусть выполнены все сделанные выше предположения, а обозначения также следуют введенным ранее. Рассмотрим дифференциальный оператор в полных производных $A: \Gamma \Omega\left(\xi_{\pi}\right) \rightarrow \varkappa(\pi)$, образ которого замкнут относительно коммутирования в алгебре $\mathfrak{g}(\pi)$ :

$$
[\operatorname{im} A, \operatorname{im} A] \subseteq \operatorname{im} A \Longleftrightarrow[A(\mathbf{p}), A(\mathbf{q})]=A\left([\mathbf{p}, \mathbf{q}]_{A}\right), \quad \mathbf{p}, \mathbf{q} \in \Gamma \Omega\left(\xi_{\pi}\right) .
$$


Оператор $A$ задает по структуре алгебры Ли $[] \mid$, im $A$ в подалгебре Ли алгебры $\mathfrak{g}(\pi)$ скобку $[,]_{A}$ на факторпространстве $\Gamma \Omega\left(\xi_{\pi}\right) / \operatorname{ker} A$.

ОПРЕДЕЛЕНИЕ 2. Тройка

$$
\left(\Gamma \Omega\left(\xi_{\pi}\right),[,]_{A}\right) \stackrel{A}{\rightarrow}(\varkappa(\pi),[,])
$$

называется вариационным алгеброидом Ли $\mathfrak{A}$ над пространством бесконечных струй $J^{\infty}(\pi)$, а гомоморфизм алгебр Ли $A-$ вариационным якорем.

По существу, вариационный якорь в вариационном алгеброиде Ли является подчиненным некоторым дополнительным условиям линейным отображением из наперед заданного горизонтального модуля сечений индуцированного расслоения над пространством бесконечных струй $J^{\infty}(\pi)$ в фиксированный горизонтальный модуль производящих сечений эволюционных дифференцирований на $J^{\infty}(\pi)$.

ПРимеР 2. Рассмотрим гамильтонов оператор

$$
A_{2}=-\frac{1}{2} \frac{d^{3}}{d x^{3}}+w \frac{d}{d x}+\frac{d}{d x} \circ w,
$$

задающий вторую пуассонову структуру уравнения КдФ $w_{t}=-w_{x x x} / 2+3 w w_{x}$. Образ оператора $A_{2}$ замкнут относительно коммутирования: скобка [ , ] $A_{2}$ равна [10]

$$
[p, q]_{A_{2}}=\partial_{A_{2}(p)}(q)-\partial_{A_{2}(q)}(p)+\frac{d}{d x}(p) \cdot q-p \cdot \frac{d}{d x}(q) .
$$

Пример 3. В работе [16] мы отмечали, что бездисперсная трехкомпонентная система Буссинеска гидродинамического типа допускает двухпараметрическое семейство конечных деформаций $[,]_{\epsilon}$ стандартной скобки $[$,$] в алгебре симметрий \operatorname{sym} \mathcal{E}$ этого уравнения. Для обоснования этого мы использовали два дифференциальных оператора рекурсии $R_{i}: \operatorname{sym} \mathcal{E} \rightarrow \operatorname{sym} \mathcal{E}, i=1,2$, образы которых замкнуты относительно коммутирования и которые, более того, совместны в указанном смысле они порождают двумерное линейное пространство операторов $R_{\epsilon}$ с инволютивными образами. Новые скобки $[,]_{\epsilon}$ получаются тогда по формуле (5) (см. статью [19] и содержащиеся в ней ссылки): $\left[R_{\boldsymbol{\epsilon}}(p), R_{\boldsymbol{\epsilon}}(q)\right]=R_{\boldsymbol{\epsilon}}\left([p, q]_{\boldsymbol{\epsilon}}\right)$, где $p, q \in \operatorname{sym} \mathcal{E}$ и $\boldsymbol{\epsilon} \in \mathbb{R}^{2} \backslash\{0\}$.

ЗАмечАниЕ 3. Как обычно, мы трактуем автономные эволюционные уравнения как $\pi$-вертикальные эволюционные векторные поля на расслоениях бесконечных струй $J^{\infty}(\pi)$ сечений векторных расслоений $\pi$ над $\Sigma^{n} \ni x$. Рассматриваемая ситуация сводится таким образом к "вырожденному" случаю пустого пространства струй.

Вообще говоря, теорема Фробениуса неверна для инволютивных распределений, порожденных образами вариационных якорей. Однако системы $\mathcal{E}$ уравнений Эйлера-Лагранжа лиувиллева типа [8], [10] являются исключением благодаря уникальной структуре своих симметрий: вариационные якори задают инволютивные распределения эволюционных векторных полей, касающихся интегральных многообразий, что неудивительно, самих уравнений $\mathcal{E}$. В таком случае (когда уравнение $\mathcal{E} \subset J^{\infty}(\pi)$ задано) мы должным образом ограничиваем определение вариационного алгеброида Ли на бесконечномерное многообразие $\mathcal{E}$ так, что в образе вариационных якорей лежат его симметрии, и, если требуется, уменьшаем слои расслоения $\xi$. 
Пример 4. Образующие алгебры Ли точечных симметрий $(2+1)$-мерного "небесного" уравнения Тоды $u_{x y}=e^{-u_{z z}}[20]$ имеют вид $\varphi^{x}=\widehat{\square}^{x}(p(x))$ либо $\varphi^{y}=\widehat{\square}^{y}(\bar{p}(y))$, где $p$ и $\bar{p}-$ произвольные гладкие функции и

$$
\widehat{\square}^{x}=u_{x}+\frac{1}{2} z^{2} \frac{d}{d x}, \quad \widehat{\square}^{y}=u_{y}+\frac{1}{2} z^{2} \frac{d}{d y} .
$$

Образ каждого из операторов замкнут относительно коммутирования так, что

$$
[p, q]_{\hat{\square}^{x}}=\partial_{\hat{\square}^{x}(p)}(q)-\partial_{\hat{\square}^{x}(q)}(p)+p \cdot \frac{d}{d x}(q)-\frac{d}{d x}(p) \cdot q
$$

для любых $p(x)$ и $q(x)$, и аналогично для $\widehat{\square}^{y}$.

Пример 5. Два особо важных примера вариационных алгеброидов Ли происходят из вариационного (ко)касательного расслоения над $J^{\infty}(\pi)$ (см. пример 1 и замечание 2). В обоих случаях их существенной составной частью оказывается подстановка Миуры, которую мы обсуждали в работе [8].

А именно, рассмотрим индуцированное расслоение $\pi_{\infty}^{*}(\zeta)$ и фиксируем какое-либо его сечение $w$. Поскольку это не вызовет никакой путаницы, мы продолжим обозначать одной и той же буквой $w$ и координаты вдоль слоя $\zeta$, и фиксированное сечение $w[u] \in \Gamma\left(\pi_{\infty}^{*}(\zeta)\right)$, которое является нелинейным дифференциальным оператором по $u$.

Очевидно, что подстановка $w=w[u]$ превращает горизонтальные $\mathcal{F}(\zeta)$-модули в подмодули горизонтальных $\mathcal{F}(\pi)$-модулей ${ }^{1)}$. Так мы получаем модули

$$
\left.\varkappa(\zeta)\right|_{w[u]: J \infty(\pi) \rightarrow \Gamma(\zeta)},\left.\quad \hat{\varkappa}(\zeta)\right|_{w[u]: J \infty(\pi) \rightarrow \Gamma(\zeta)},
$$

понимая последний как модуль сечений расслоения, индуцированного с помощью $\pi_{\infty}^{*}$ из расслоения, двойственного к $\zeta_{\infty}^{*}(\zeta)$ относительно спаривания со значениями в $\bar{\Lambda}^{n}(\zeta)$. Обратим особое внимание, что с помощью именно этого построения нам удается сохранить истинные правила преобразования для сечений, лежащих в $\varkappa(\zeta)$ или в $\hat{\varkappa}(\zeta)$, при (никак не связанных между собой!) заменах координат вдоль слоя расслоений $\zeta$ и $\pi$, т. е. соответственно координат $w$ и $u$.

Будем говорить, что линейные операторы $A:\left.\varkappa(\zeta)\right|_{w[u]} \rightarrow \varkappa(\pi)$ и $A:\left.\widehat{\varkappa}(\zeta)\right|_{w[u]} \rightarrow$ $\varkappa(\pi)$, наделенные свойством (5), являются вариационными якорями первого и второго типа соответственно. При дифференциальных заменах $\tilde{u}=\tilde{u}[u]: J^{\infty}(\pi) \rightarrow$ $\Gamma(\pi)$ и $\widetilde{w}=\widetilde{w}[w]: J^{\infty}(\zeta) \rightarrow \Gamma(\zeta)$ операторы $A$ первого типа преобразуются по формуле

$$
A \mapsto \tilde{A}=\left.\ell_{\tilde{u}}^{(u)} \circ A \circ \ell_{w}^{(\widetilde{w})}\right|_{w=w[u], u=u[\tilde{u}]} .
$$

Операторы второго типа подчинены правилу преобразования

$$
A \mapsto \tilde{A}=\left.\ell_{\tilde{u}}^{(u)} \circ A \circ\left(\ell_{\tilde{w}}^{(w)}\right)^{\dagger}\right|_{w=w[u], u=u[\tilde{u}]},
$$

где † означает взятие сопряженного оператора. Операторы рекурсии с инволютивными образами, рассмотренные нами в работе [16], служат примерами якорей первого типа. Все гамильтоновы операторы и, в более общем случае, нётеровы операторы

\footnotetext{
1) $\mathrm{K}$ примеру, для операторов рекурсии $\varkappa(\pi) \rightarrow \varkappa(\pi)$ имеем $\zeta=\pi$ (см. пример 3$)$. По умолчанию область определения гамильтоновых операторов обычно отождествляется с модулем $\hat{\varkappa}(\pi)$ (см. пример 2). В обоих случаях мы полагаем $w=\mathrm{id}: \Gamma(\pi) \rightarrow \Gamma(\zeta)$; именно поэтому вспомогательное расслоение $\zeta$ остается "невидимым" в стандартном изложении (например, в работах [18], [21]).
} 
с инволютивными образами являются вариационными якорями второго типа [16]. Операторы $\square$ и $\square$, задающие симметрии систем уравнений Эйлера-Лагранжа лиувиллева типа, также относятся к якорям второго типа; именно в этом смысле они оказываются “некососопряженными обобщениями гамильтоновых операторов" [10].

В оставшейся части данного раздела мы описываем каноническое разложение скобки $[,]_{A}$, индуцированной на факторпространстве $\Gamma \Omega\left(\xi_{\pi}\right)$ по модулю ker $A$. По правилу Лейбница в скобке эволюционных векторных полей $A(\mathbf{p})$ и $A(\mathbf{q})$, принадлежащих образу вариационного якоря $A$, возникают два набора слагаемых:

$$
[A(\mathbf{p}), A(\mathbf{q})]=A\left(\partial_{A(\mathbf{p})}(\mathbf{q})-\partial_{A(\mathbf{q})}(\mathbf{p})\right)+\left(\partial_{A(\mathbf{p})}(A)(\mathbf{q})-\partial_{A(\mathbf{q})}(A)(\mathbf{p})\right)
$$

В первой паре слагаемых мы использовали перестановочность эволюционных дифференцирований с полными производными. Вторая пара слагаемых обязана попасть в образ оператора $A$ по построению. Следовательно, структура алгебры Ли $[,]_{A}$ на области определения оператора $A$ приобретает вид

$$
[\mathbf{p}, \mathbf{q}]_{A}=\partial_{A(\mathbf{p})}(\mathbf{q})-\partial_{A(\mathbf{q})}(\mathbf{p})+\{\{\mathbf{p}, \mathbf{q}\}\}_{A}
$$

Итак, скобка $[,]_{A}$, определенная с точностью до $\operatorname{ker} A$, состоит из двух стандартных слагаемых и бидифференциальной кососимметричной компоненты $\{\{,\}\}_{A} \in$ $\mathcal{C} \operatorname{Diff}\left(\Gamma \Omega\left(\xi_{\pi}\right) \times \Gamma \Omega\left(\xi_{\pi}\right) \rightarrow \Gamma \Omega\left(\xi_{\pi}\right)\right)$. При любых $\mathbf{p}, \mathbf{q}, \mathbf{r} \in \Gamma \Omega\left(\xi_{\pi}\right)$ тождество Якоби для скобки (9) имеет вид

$$
\begin{aligned}
0= & \sum_{\circlearrowright}\left[[\mathbf{p}, \mathbf{q}]_{A}, \mathbf{r}\right]_{A}=\sum_{\circlearrowright}\left[\partial_{A(\mathbf{p})}(\mathbf{q})-\partial_{A(\mathbf{q})}(\mathbf{p})+\{\{\mathbf{p}, \mathbf{q}\}\}_{A}, \mathbf{r}\right]_{A}= \\
= & \sum_{\circlearrowright}\left\{\partial_{A\left(\partial_{A(\mathbf{p})}(\mathbf{q})-\partial_{A(\mathbf{q})}(\mathbf{p})\right)}(\mathbf{r})-\partial_{A(\mathbf{r})}\left(\partial_{A(\mathbf{p})}(\mathbf{q})-\partial_{A(\mathbf{q})}(\mathbf{p})\right)+\right. \\
& +\left\{\left\{\partial_{A(\mathbf{p})}(\mathbf{q})-\partial_{A(\mathbf{q})}(\mathbf{p}), \mathbf{r}\right\}\right\}_{A}+ \\
& \left.+\partial_{A\left(\{\{\mathbf{p}, \mathbf{q}\}\}_{A}\right)}(\mathbf{r})-\underline{\partial_{A(\mathbf{r})}\left(\{\{\mathbf{p}, \mathbf{q}\}\}_{A}\right)}+\left\{\left\{\{\{\mathbf{p}, \mathbf{q}\}\}_{A}, \mathbf{r}\right\}\right\}_{A}\right\} .
\end{aligned}
$$

Подчеркнутый член содержит дифференцирования коэффициентов скобки $\{\{,\}\}_{A}$, принадлежащих кольцу $\mathcal{F}(\pi)$. Отметим, что даже если результат применения эволюционных полей $\partial_{\varphi}^{(u)}=\varphi \partial / \partial u+\cdots$ к аргументам оператора $A$ положен равным нулю (что имеет смысл, см. ниже), то эти слагаемые могут оказаться отличными от нуля. При этом тождество Якоби для $[,]_{A}$ вырождается в равенство

$$
\sum_{\circlearrowright}\left(-\partial_{A(\mathbf{r})}^{(\mathbf{u})}\left(\{\{\mathbf{p}, \mathbf{q}\}\}_{A}\right)+\left\{\left\{\{\{\mathbf{p}, \mathbf{q}\}\}_{A}, \mathbf{r}\right\}\right\}_{A}\right)=0 .
$$

Формулы (5) и (9), формула (10), а также $\left(10^{\prime}\right)$ сыграют в следующем разделе такую же роль в доказательстве приведенной ниже теоремы, какая была отведена соответственно лемме 1, формулам (4), а также (1) (либо (3)) при доказательстве эквивалентности двух классических определений. 


\section{3. ГОМОЛОГИЧЕСКИЕ ЭВОЛЮЦИОННЫЕ ВЕКТОРНЫЕ ПОЛЯ}

В этом разделе мы строим представление вариационных алгеброидов Ли в терминах гомологических эволюционных векторных полей $Q$ на суперрасслоениях бесконечных струй; эти суперрасслоения мы естественным образом связываем с определением 2. Нам потребуется сделать два подготовительных шага. Во-первых, предстоит установить, какой объект является нечетным соседом ${ }^{2)} \overline{J^{\infty}}\left(\Pi \xi_{\pi}\right)$ горизонтального расслоения бесконечных струй $\bar{J}\left(\xi_{\pi}\right)$ над $J^{\infty}(\pi)$. Во-вторых, на вариационные якори $A$ будут наложены условия, чтобы получаемое в итоге гомологическое поле $Q$ стало корректно определенным на $\bar{J}{ }^{\infty}\left(\Pi \xi_{\pi}\right)$; для этого мы применим технику, уже испытанную в работе [8].

Итак, напомним, что по построению расслоение $\xi_{\pi}: \Omega\left(\xi_{\pi}\right) \rightarrow J^{\infty}(\pi)$ индуцировано с помощью $\pi_{\infty}$ из векторного расслоения $\xi$ над базой $\Sigma^{n}$. Рассмотрим горизонтальное расслоение бесконечных струй $\overline{J^{\infty}}\left(\xi_{\pi}\right)$ сечений векторного расслоения $\pi_{\infty}^{*}(\xi)$ над $J^{\infty}(\pi)$ (подробности и примеры см. в работах [12], [18], [21]). Теперь обратим четность слоев расслоения $\overline{J^{\infty}}\left(\xi_{\pi}\right) \rightarrow J^{\infty}(\pi)$ и введем обозначение $\Pi: \mathbf{p} \mapsto \mathbf{b}$ для смены четности; о переменных $\mathbf{p}$ и $\mathbf{b}$ сказано чуть ниже. Таким образом мы получаем горизонтальное суперрасслоение бесконечных струй над $J^{\infty}(\pi)$, обозначаемое в дальнейшем через $\overline{J^{\infty}}\left(\Pi \xi_{\pi}\right)$. Подытожим введенные ранее обозначения:

- $x$ - набор $n$ локальных координат на базе, многообразии $\Sigma^{n}$;

- $u_{\boldsymbol{\sigma}}$, где $|\boldsymbol{\sigma}| \geqslant 0,-$ струйные координаты в слое расслоения $\pi_{\infty}: J^{\infty}(\pi) \rightarrow \Sigma^{n}$;

- $\mathbf{p}$ - координата вдоль слоя индуцированного расслоения $\pi_{\infty}^{*}(\xi)$ над $J^{\infty}(\pi)$, a $\mathbf{p}, \mathbf{p}_{x}, \ldots, \mathbf{p}_{\boldsymbol{\tau}}$ при произвольном мультииндексе $\boldsymbol{\tau}$ - координаты вдоль слоя в горизонтальном расслоении бесконечных струй $\overline{J^{\infty}}\left(\xi_{\pi}\right)$ сечений этого индуцированного расслоения;

$\bullet \mathbf{b}, \mathbf{b}_{x}, \ldots, \mathbf{b}_{\boldsymbol{\tau}}$, где $|\boldsymbol{\tau}| \geqslant 0$, - нечетные координаты вдоль слоя горизонтального суперрасслоения бесконечных струй $\overline{J^{\infty}}\left(\Pi \xi_{\pi}\right) \rightarrow J^{\infty}(\pi)$;

- вариационный якорь $A$, будучи линейным дифференциальным оператором в полных производных, тем самым является функцией, линейной по слоям - как расслоения $\overline{J^{\infty}}\left(\xi_{\pi}\right)$, так и суперрасслоения $\overline{J^{\infty}}\left(\Pi \xi_{\pi}\right)-$ и принимающей значения в пространстве производящих сечений эволюционных векторных полей на $J^{\infty}(\pi)$;

- $[,]_{A}$ - скобка (9) на горизонтальном модуле $\Gamma \Omega\left(\xi_{\pi}\right)$; она определена с точностью до ядра оператора $A$; выберем любой представитель бидифференциальной операции

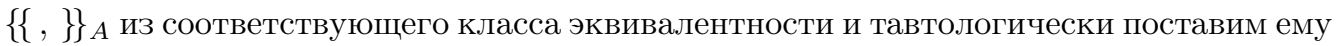
в соответствие билинейную функцию на горизонтальном пространстве струй.

Расположенный над каждой точкой $\theta^{\infty} \in J^{\infty}(\pi)$ нечетный слой горизонтального суперрасслоения бесконечных струй $\overline{J^{\infty}}\left(\Pi \xi_{\pi}\right)$ содержит линейное подпространство $\operatorname{ker} A$, определенное линейным уравнением $\left.A\right|_{\theta \infty}(\mathbf{b})=0$ (точнее, его бесконечным продолжением). Важно, что линейное пространство его решений не зависит от точки [b] слоя, ибо дифференциальный оператор $\left.A\right|_{\theta^{\infty}}$ может зависеть лишь от точки базы $\theta^{\infty} \in J^{\infty}(\pi)$, а уравнение линейное. Чтобы запретить возможность параметризации ядра оператора $A$ произвольными функциями, мы требуем, чтобы оператор $A$ был невырожденным в смысле определения, примененного в работе [8]: $A \circ \nabla=0$ влечет $\nabla=0$.

${ }^{2)}$ По определению, данному Ю. И. Маниным, соседи алгебры Ли $\mathfrak{g}-$ это $\mathfrak{g}^{*}, \Pi \mathfrak{g}$ и $\mathfrak{g}^{*}$, где П функтор смены четности (см. [5]). 
ТЕорема. Пусть А-невырожденный вариационный якорь и пусть выполнены все сделанные ранее предположения и зафиксированы обозначения. Каким быни был выбор скобки $\{\{,\}\}_{\text {A }}$ по модулю $\operatorname{ker} A$, следующее нечетное эволючионное векторное поле $Q$ на горизонтальном суперрасслоении бесконечных струй $\overline{J^{\infty}}\left(\Pi \xi_{\pi}\right)$ является гомологическим:

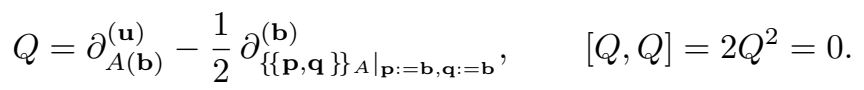

Более того, либо в классе эквивалентности $Q\left(\bmod \partial_{\mathbf{n}}^{(\mathbf{b})}\right)$ таких полей на слое расслоения $\overline{J^{\infty}}\left(\Pi \xi_{\pi}\right) \rightarrow J^{\infty}(\pi)$ над каждой точкой $\theta^{\infty} \in J^{\infty}(\pi)$ существует единственный канонический представитель (здесъ $\mathbf{n} \in \operatorname{ker} A, \operatorname{ma\kappa }$ что $\partial_{\mathbf{n}}^{(\mathbf{b})}$ - симметрия ядра), либо, если якорь А близок к вырожденному в уточняемом ниже смысле, этой единственности можно достичь, фиксируя значения скобки $\{\{,\}\}_{A}$ на (не более чем) границе звездной области, центрально-симметричной относительно нуля в слое над $\theta^{\infty}$ и содержащейся в горизонталъном расслоении струй $\overline{J|\boldsymbol{\tau}|}\left(\Pi \xi_{\pi}\right)$ некоторого конечного порядка $|\boldsymbol{\tau}|<\infty$.

Пример 6. Второй гамильтонов оператор $A_{2}$ для уравнения КдФ (см. выше) задает гомологическое векторное поле $Q=\partial_{A_{2}(b)}^{(u)}+\partial_{b b_{x}}^{(b)}$. Это простейший пример, содержащий не равный тождественно нулю дифференциальный полином второй степени от антикоммутирующих переменных b (в данном случае такая переменная всего одна) и их (соответственно ее) производных.

ДокАЗАтельство теоремы. Антикоммутатор $[Q, Q]=2 Q^{2}$ нечетного эволюционного векторного поля сам является эволюционным векторным полем. Поэтому в нем достаточно вычислить коэффициенты при $\partial / \partial \mathbf{u}$ и $\partial / \partial \mathbf{b}$; по построению, все остальные слагаемые будут содержать соответствующие полные производные этих двух коэффициентов.

Сначала найдем нечетную скорость переменной $\mathbf{u}$; она равна

$$
\partial_{A(\mathbf{b})}^{(\mathbf{u})}(A)(\mathbf{b})-\left.\frac{1}{2} A\left(\{\{\mathbf{p}, \mathbf{q}\}\}_{A}\right)\right|_{\mathbf{p}:=\mathbf{b}, \mathbf{q}:=\mathbf{b}}
$$

Используя то, что переменная b нечетная, удвоим уменьшаемое и перепишем (12) каK

$$
\left.\frac{1}{2}\left(\partial_{A(\mathbf{p})}^{(\mathbf{u})}(A)(\mathbf{q})-\partial_{A(\mathbf{q})}^{(\mathbf{u})}(A)(\mathbf{p})-A\left(\{\{\mathbf{p}, \mathbf{q}\}\}_{A}\right)\right)\right|_{\mathbf{p}:=\mathbf{b}, \mathbf{q}:=\mathbf{b}}
$$

По определению операции $\{\{,\}\}_{A}$, данному в (8), (9), выражение в скобках равно нулю.

Одновременно с этим коэффициент при $\partial / \partial \mathbf{b}$ равен

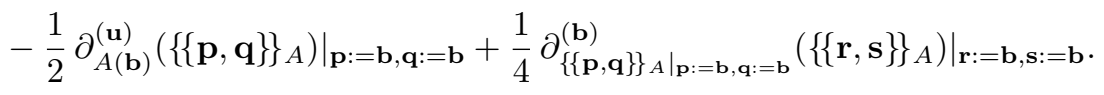

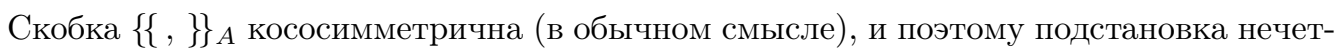
ной переменной $\mathbf{b}$ вместо каждого из аргументов удвоит ее. Заметим также, что нечетное дифференцирование $\partial_{\{\{\mathbf{p}, \mathbf{q}\}\}_{A}}^{(\mathbf{b})}$ с подставленными $\mathbf{p}:=\mathbf{b}, \mathbf{q}:=\mathbf{b}$ действует 
на аргумент с учетом градуированного правила Лейбница. По определению дифференцирование заменяет всякую производную переменной $\mathbf{b}$ соответствующей производной величины $\{\{\mathbf{b}, \mathbf{b}\}\}_{A}$. Получается, что коэффициент (13) равен

$$
\left.\frac{1}{2}\left(-\partial_{A(\mathbf{r})}^{(\mathbf{u})}\left(\{\{\mathbf{p}, \mathbf{q}\}\}_{A}\right)+\left\{\left\{\{\{\mathbf{p}, \mathbf{q}\}\}_{A}, \mathbf{r}\right\}\right\}_{A}\right)\right|_{\mathbf{p}:=\mathbf{b}, \mathbf{q}:=\mathbf{b}, \mathbf{r}:=\mathbf{b}} .
$$

Утроим это выражение, беря сумму по циклическим перестановкам $\mathbf{p} \rightarrow \mathbf{q} \rightarrow \mathbf{r} \rightarrow \mathbf{p}$, не меняющим знак. В итоге мы получаем умноженное на $1 / 6$ тождество Якоби $\left(10^{\prime}\right)$. Первое утверждение теоремы доказано.

Выше мы показали, что равенство $Q^{2}=0$ выполнено при любом выборе скобки $\{\{,\}\}_{A}+\mathbf{n}([\mathbf{b}])$, т. е. при произвольном $\mathbf{n} \in \operatorname{ker} A$. С одной стороны, линейное подпространство $\operatorname{ker} A$ в слое горизонтального расслоения струй, расположенном над точкой $\theta^{\infty} \in J^{\infty}(\pi)$, не зависит от точки [b] этого слоя. С другой стороны, представитель $\mathbf{n} \in \operatorname{ker} A$ может оказаться отличным от нуля и, вообще говоря, может зависеть от точки. Мы утверждаем, что $\mathbf{n}$ можно тривиализовать (или по крайней мере нормировать корректно определенным способом) одновременно во всех точках слоя.

Прежде всего, с помощью подходящего сдвига вдоль ядра подчиним скобку $\{\{,\}\}_{A}$ условию $\{\{[0],[0]\}\}_{A} \equiv 0$, наложенному в нуле $[\mathbf{b}]=0$ векторного пространства - слоя над точкой $\theta^{\infty} \in J^{\infty}(\pi)$ в векторном расслоении $\overline{J^{\infty}}\left(\Pi \xi_{\pi}\right) \rightarrow J^{\infty}(\pi)$. Теперь заметим, что оставшийся “довесок" $\mathbf{n}$, во всех точках слоя принадлежащий общему для них ядру $\operatorname{ker} A$, является квадратично-однородным по координатам $[\mathbf{b}]$ горизонтальной струи, иными словами, $\mathbf{n}=\mathbf{n}([\mathbf{b}] \cdot[\mathbf{b}])$. Условие невырожденности оператора $A$ исключает возможность появления произвольной функциональной зависимости от $[\mathbf{b}] \cdot[\mathbf{b}]$ у сечений, принадлежащих области определения какого-либо не равного нулю оператора $\nabla$, такого что $A \circ \nabla \equiv 0$.

Дифференциальный порядок скобки $\{\{,\}\}_{A}$ конечен и не превосходит суммы дифференциальных порядков собственно оператора $A$ и его коэффициентов, которые могут зависеть от $[\mathbf{u}]$. Таким образом, $\mathbf{n}=\mathbf{n}\left(\left(\mathbf{b}, \ldots, \mathbf{b}_{\boldsymbol{\tau}}\right) \cdot\left(\mathbf{b}, \ldots, \mathbf{b}_{\boldsymbol{\tau}}\right)\right)$, причем $|\boldsymbol{\tau}|<\infty$. Возьмем нечетные переменные $\mathbf{b}, \ldots, \mathbf{b}_{\boldsymbol{\tau}}$ и рассмотрим все те попарные произведения $\psi={ }^{\mathrm{t}}\left(b^{i} \cdot b^{j}, \ldots, b_{\boldsymbol{\tau}}^{i} \cdot b_{\boldsymbol{\tau}}^{j}\right)$, которые не равны нулю тождественно. Применяя метод неопределенных коэффициентов, составим из них $m$-компонентный столбец $\mathbf{n}=\nabla(\psi)$ : пусть $\nabla_{\beta}^{\alpha} \in \mathbb{R}$ и $\mathbf{n}^{\alpha}=\nabla_{\beta}^{\alpha} \cdot \psi^{\beta}$. Предположение о том, что $\mathbf{n} \neq 0$, формально не противоречит нашему исходному предположению о невырожденности оператора $A$, поскольку полученное таким способом сечение $\psi$ не является произвольным (может оказаться, что столбец задан меньшим числом параметров, чем число компонент в нем, из-за наличия полиномиальных соотношений между этими компонентами). Если отличных от нуля матриц $\nabla$ не существует, доказательство окончено. Предположим, однако, что решение $\nabla \neq 0$ все-таки нашлось. Тогда при растяжении слоя в $\lambda \in \mathbb{R}$ раз отклонение $\mathbf{n}(\lambda) \in \operatorname{ker} A$ от нормировки $\mathbf{n} \equiv 0$ в нуле будет возрастать квадратично по $\lambda$. Следовательно, чтобы однозначно задать скобку $\{\{,\}\}_{A}$, достаточно зафиксировать значения “довеска" $\mathbf{n}$ в точках границы центрально-симметричной звездной области с центром в нуле, расположенной над точкой $\theta^{\infty}$ в конечномерном слое пространства конечных струй $\overline{J^{|\tau|}}\left(\Pi \xi_{\pi}\right)$. С помощью проведенного выше рассуждения мы нормировали $\{\{,\}\}_{A}$ на всем слое расслоения $\overline{J^{\infty}}\left(\Pi \xi_{\pi}\right)$ и тем самым определили канонический представитель $Q$ в классе полей (11) на этом слое. Доказательство теоремы завершено. 
ЗАМЕчАНИЕ 4. Мы начали доказательство с того, что использовали постулированное свойство вариационного якоря $A$ быть гомоморфизмом алгебр Ли. Последующее рассуждение основано на кососимметричности операции $\{\{,\}\}_{A}$, на (всегда выполненном для дифференцирований) правиле Лейбница и на тождестве Якоби для скобки $[,]_{A}$. В каком-то смысле слагаемые в формуле $\left(10^{\prime}\right)$ можно трактовать как правую часть нового "правила Лейбница" для скобки (9) в вариационном алгеброиде Ли.

ЗАмЕчАниЕ 5. Гомологическое эволюционное векторное поле (11) не содержит свободного члена и квадратично по нечетной переменной $\mathbf{b}$ и ее производным. Допустим, что эта степень полинома есть произвольное положительное число (быть может, даже бесконечность), а все остальные предположения по-прежнему выполнены. Таким образом получаются гомотопические деформации Шлезингера-Сташефа [22] структуры алгебры Ли в $\left(\Gamma \Omega\left(\xi_{\pi}\right),[,]_{A}\right)$. В частности, тождество Якоби $[Q, Q]=0$ вовлекает теперь не только двухместную скобку $[,]_{A}$, но также и трех-, четырех- и прочие $N$-местные кососимметричные скобки с числом аргументов $N \geqslant 3$ (см. [2], [5], [22], [23] и ссылки в этих работах).

В статье [24] (см. также ссылки в ней) утверждалось, что при $N \geqslant 3$ не существуют вариационные $N$-векторы для всех эволюционных систем дифференциального порядка выше первого и с обратимым символом ${ }^{3)}$. Из этого утверждения следует жесткость относительно гомотопий структуры вариационных алгеброидов Ли, задаваемой гамильтоновыми операторами для таких уравнений.

Вариационное мастер-уравнение: иллюстрация. Рассмотрим более подробно свойства гомологического векторного поля (11) в частном случае, когда якорь $A: \hat{\varkappa}(\pi) \rightarrow \varkappa(\pi)$ - гамильтонов дифференциальный оператор и, таким образом, его аргументами $\mathbf{p}$ являются вариационные ковекторы ${ }^{4)}$. В этом случае индуцированная скорость $\mathbf{p}$ известна (см. ниже формулу (14)), если задана эволюция $\dot{u}=A(\mathbf{p})$. Соответствующее эволюционное векторное поле (11) на горизонтальном суперрасслоении струй $\overline{J^{\infty}}\left(\Pi \widehat{\pi}_{\pi}\right)$ было построено для гамильтоновых операторов $A$ в недавней работе [21] именно с этих позиций: коэффициент при $\partial / \partial \mathbf{b}$ был постулирован раз и навсегда в соответствии с формулой (14) и не подлежал в дальнейшем интерпретации как скобка $\{\{\mathbf{b}, \mathbf{b}\}\}_{A}$. В рамках такого подхода квадратное уравнение $Q^{2}=0$, неизвестные в котором суть кососопряженные операторы $A$, задает вариационные пуассоновы структуры.

В рассматриваемом здесь вариационном пуассоновом случае гомологическое векторное поле (11) можно вычислить явно для каждого гамильтонова оператора $A$.

\footnotetext{
3) Как легко видеть, второе условие зависит от выбора локальных координат. Мы полагаем, что то же утверждение все же остается справедливым для эволюционных систем дифференциального порядка выше первого и с невырожденными линеаризациями правой части.

4) По определению вариационными ковекторами называются гомоморфизмы, действующие из пространства $\varkappa(\pi)=\Gamma\left(\pi_{\infty}^{*}(\pi)\right)$ производящих сечений $\varphi$ эволюционных дифференцирований $\partial_{\varphi}$ в пространство горизонтальных дифференциальных форм степени $n$ на тотальном пространстве $J^{\infty}(\pi)$ расслоения $\pi_{\infty}$ над $\Sigma^{n}$. Эта конструкция неявно предписывает выбрать форму объема $d \mathbf{x}$ на базе и тем самым фиксировать спаривание $\langle$,$\rangle между вариационными ковекторами$ и эволюционными векторными полями. С этой точки зрения гомологическое векторное поле $Q$, заданное гамильтоновым оператором $A$, является полем Баталина-Вылковысского, а не просто БРСТ-полем [2].
} 
Более того, поле $Q$ само оказывается гамильтоновым относительно вариационного пуассонова бивектора $\mathbf{H}=A(\mathbf{b}) \cdot \mathbf{b} / 2$ и канонической симплектической структуры [13], [25] на горизонтальном суперпространстве бесконечных струй $\overline{J^{\infty}}\left(\Pi \widehat{\pi}_{\pi}\right)$. Ниже мы иллюстрируем этот стандартный алгебраический факт, используя технику, изложенную в обзоре [12].

Скобка $\{\{,\}\}_{A}$, заданная гамильтоновым оператором $A$, получается, если расписать тождество Якоби для структуры алгебры Ли $\left(\bar{H}^{n}(\pi),\{,\}_{A}\right)$ гамильтоновых функционалов, наделенных при помощи оператора $A$ скобкой Пуассона. Следуя обозначениям книги [26], мы положим $\ell_{A, \psi}^{(\mathbf{u})}(\varphi):=\left(\partial_{\varphi}(A)\right)(\psi)$ для произвольных $\varphi \in \varkappa(\pi), \psi \in \hat{\varkappa}(\pi)$ и дифференциального оператора в полных производных $A \in \mathcal{C} \operatorname{Diff}(\hat{\varkappa}(\pi), \varkappa(\pi))$. Отметим, что $\ell_{A, \psi}^{(\mathbf{u})}$ является оператором в полных производных по отношению к своему аргументу $\varphi$ и по отношению к $\psi$ (но не к коэффициентам оператора $A$ ), и потому корректно определен сопряженный оператор $\left(\ell_{A, \psi}^{(\mathbf{u})}\right)^{\dagger}$.

Лемма 3 [16], [26]. Кососопряженный оператор А является гамильтоновым тогда и толъко тогда, когда для всех $\mathbf{p}, \mathbf{q} \in \hat{\varkappa}(\pi)$ выполнено соотношение

$$
\left(\partial_{A(\mathbf{p})}(A)\right)(\mathbf{q})-\left(\partial_{A(\mathbf{q})}(A)\right)(\mathbf{p})=A\left(\left(\ell_{A, \mathbf{p}}^{(\mathbf{u})}\right)^{\dagger}(\mathbf{q})\right) .
$$

Вследствие этого

$$
\{\{\mathbf{p}, \mathbf{q}\}\}_{A}=\left(\ell_{A, \mathbf{p}}^{(\mathbf{u})}\right)^{\dagger}(\mathbf{q}), \quad \mathbf{p}, \mathbf{q} \in \widehat{\varkappa}(\pi),
$$

если $A$ - гамильтонов оператор; с другой стороны, условие (14) задает удобный критерий, позволяющий проверить, является ли гамильтоновым данный дифференииальный оператор в полных производных.

Следует уточнить, что $\ell_{A, \mathbf{p}}^{(\mathbf{u})}$ - это не то же самое, что линеаризация $\ell_{A(\mathbf{p})}^{(u)}$. Однако если $\mathbf{p}$ становится струйной координатой в горизонтальном расслоении струй над $J^{\infty}(\pi)$, то эти два обозначения превращаются в синонимы (см., например, [21]). Более того, тогда несложно показать, что имеют место равенства ${ }^{5)}$

$$
\frac{1}{2}\left(\ell_{A, \mathbf{b}}^{(\mathbf{u})}\right)^{\dagger}(\mathbf{b})=\frac{\delta}{\delta \mathbf{u}}\left(\frac{1}{2} A(\mathbf{b}) \cdot \mathbf{b}\right), \quad A(\mathbf{b})=\frac{\delta}{\delta \mathbf{b}}\left(\frac{1}{2} A(\mathbf{b}) \cdot \mathbf{b}\right) ;
$$

второе тождество выполнено вследствие того, что гамильтонов оператор $A$ является кососопряженным. В заключение напомним факт, известный из работ [13], [25]: нечетный "сосед" $\overline{J^{\infty}}\left(\Pi \widehat{\pi}_{\pi}\right) \rightarrow J^{\infty}(\pi)$ вариационного кокасательного расслоения наделен канонической симплектической структурой $\boldsymbol{\omega}=\left(\begin{array}{cc}0 & 1 \\ -1 & 0\end{array}\right)$. Уточним, что структура $\boldsymbol{\omega}$ присутствует, даже если пространство значений для локальных сечений расслоения $\pi$ или, в более общем случае, многообразие образов, в котором принимают значения отображения $\mathbf{u}: \Sigma^{n} \rightarrow M^{m}$, само по себе не является симплектическим. В противоположность этому в статье [2] симплектическая структура на пространстве отображений $\mathcal{E}=\left\{\Sigma^{n} \rightarrow M^{m}\right\}$ была построена по 2-форме на $M^{m}$, т. е. с необходимостью предполагалось, что четномерное многообразие образов является симплектическим.

\footnotetext{
5) По соглашению $\delta S=(\delta S / \delta \eta) \delta \eta$ все вариации $\delta \eta$ переменных $\eta$ (быть может, нечетных) пропускаются до упора вправо, что прискорбно, поскольку это полностью рассогласуется с идеей Дирака располагать в формулах bra-ковекторы слева и ket-векторы справа (в частности, в скобках "bra-c-ket") и вносит в формулы дополнительные знаки труднообъяснимой природы.
} 
Подытожим накопленный результат. Он отражает, теперь уже в рамках вариационной картины, свойства гомологических векторных полей $Q$, установленные в работе [2] для конечномерного случая.

УтвЕРЖДЕНИЕ. Для каждого гамильтонова дифференииалъного оператора $А$ гомологическое эволючионное векторное поле (11) само является гамильтоновым относительно вариационного пуассонова бивектора $\mathbf{H}=A(\mathbf{b}) \cdot \mathbf{b} / 2$ и канонической симплектической структуры $\boldsymbol{\omega}$ :

$$
Q=\partial_{\delta \mathbf{H} / \delta \mathbf{b}}^{(\mathbf{u})}+\partial_{-\delta \mathbf{H} / \delta \mathbf{u}}^{(\mathbf{b})}
$$

Лагранжиан $\mathbf{L}=\mathbf{\mathbf { u }} \mathbf{-} \mathbf{H}$, полученный преобразованием Лежандра из гамильтониана $\mathbf{H}$, равен $\mathbf{L}=A(\mathbf{b}) \cdot \mathbf{b} / 2$ и удовлетворяет вариационному мастер-уравнению $\llbracket \mathbf{L}, \mathbf{L} \rrbracket=0$, где $\llbracket$, - вариационная скобка Схоутена.

ПримеР 7. Вновь рассмотрим гамильтонов оператор

$$
A_{2}=-\frac{1}{2} \frac{d^{3}}{d x^{3}}+w \frac{d}{d x}+\frac{d}{d x} \circ w
$$

для уравнения КдФ; скобка (14) равна $\{\{p, q\}\}_{A_{2}}=p_{x} q-p q_{x}$ (см. (7)). Вариационный пуассонов бивектор получается таким: $\mathbf{H}=-b_{x x x} b / 4+w b_{x} b$, откуда (см. сноску 5 )

$$
\frac{\delta \mathbf{H}}{\delta b}=-\frac{1}{2} b_{x x x}+2 w b_{x}+w_{x} b, \quad \frac{\delta \mathbf{H}}{\delta w}=b_{x} b .
$$

Гомологическое эволюционное векторное поле (11) равно $Q=\partial_{A_{2}(b)}^{(w)}-\partial_{2 b_{x} b}^{(b)} / 2$, что в точности совпадает с выражением (15).

В заключение отметим, что явно заданныйб) гамильтониан $\mathbf{H}$ поля $Q$ никак не связан с гамильтонианом $\mathcal{H}$ наблюдаемого движения $\dot{\mathbf{u}}=A(\delta \mathcal{H} / \delta \mathbf{u})$ образа многообразия $\Sigma^{n}$ по многообразию $M^{m}$, так как гамильтониан $\mathcal{H}$ может вообще не существовать!

\section{4. ВЫВОДЫ}

Мы видим, что мотивированная физическими приложениями геометрия, рассматривавшаяся в статье [2], имеет явно вариационную природу. Иначе говоря, модель струны $\Sigma^{n} \rightarrow M^{m}$ обязывает нас использовать расслоения струй, которые заключают в себе данные не только об одних лишь отображениях, но и обо всех их производных. Можно пытаться задавать разнообразные конструкции нулевого дифференциального порядка относительно многообразия $\Sigma^{n}$, в частности пуассоновы структуры или гомологические векторные поля, но полная картина при этом учтена не будет. В физических терминах равенство нулю всех производных означает, что либо $n=0$ и $\Sigma=\{\mathrm{pt}\}$ (т. е. не по чему дифференцировать), либо образ многообразия $\Sigma^{n}$ при постоянном отображении становится точкой в пространстве $M^{m}$, и возникающая таким образом точечная частица наделена $n$ скрытыми степенями

6) Лагранжиан $\mathbf{L}$ и гамильтониан $\mathbf{H}$ совпадают, и потому вариационный пуассонов формализм можно рассматривать как (весьма отдаленный) раздел теории равномерного прямолинейного движения. 
свободы. В настоящей работе мы вводили определения и устанавливали их свойства, в целом следуя подходу статьи [1]; таким образом нам удалось снять ограничение, присущее рассуждениям из работы [2], состоявшее в том, что частицы реально могли быть лишь точечными. Более того, теперь видно, что еще одно предположение, сделанное в работе [2], - о наличии симплектической структуры на многообразии образов $M^{m}$ - избыточно для того, чтобы гомологическое эволюционное поле Баталина-Вылковысского было гамильтоновым относительно канонической симплектической структуры на пространстве отображений (хотя, конечно же, вариационный подход остается применимым к изучению пуассоновых сигма-моделей). Мы надеемся, что изложенные здесь соображения окажутся полезными при исследовании не требующих размерной редукции струнных моделей в пространстве-времени Минковского $M^{3,1}$.

Благодарности. Авторы благодарны Б.А. Дубровину, И. С. Красильщику, М. А. Нестеренко, П. Дж. Олверу, В. Н. Рубцову, В. В. Соколову за обсуждения и высказанные замечания. А. В. Киселев признателен организационному комитету конференции "Нелинейная физика: теория и эксперимент. VI" за радушное гостеприимство. А. В. Киселев также благодарит IHÉS, SISSA и CRM (Montréal, Canada) за финансовую поддержку и гостеприимство. Работа выполнена при частичной финансовой поддержке со стороны Европейского Союза (FP6 Marie Curie RTN ENIGMA, контракт MRTN-CT-2004-5652), European Science Foundation Program MISGAM, a также NWO (гранты B61-609 и VENI 639.031.623).

\section{Список литературы}

[1] А. Ю. Вайнтроб, УМН, 52:2(314) (1997), 161-162.

[2] M. Alexandrov, A. Schwarz, O. Zaboronsky, M. Kontsevich, Internat. J. Modern Phys. A, 12:7 (1997), 1405-1429, arXiv: hep-th/9502010.

[3] Y. Kosmann-Schwarzbach, SIGMA, 4 (2008), 005, 30 pp., arXiv:0710.3098; Lett. Math. Phys., 69:1-3 (2004), 61-87, arXiv: math/0312524; Ann. Inst. Fourier (Grenoble), 46:5 (1996), 1243-1274.

[4] А. В. Бочаров, А. М. Вербовецкий, А. М. Виноградов, С. В. Дужин, И. С. Красильщик, А. В. Самохин, Ю. Н. Торхов, Н. Г. Хорькова, В. Н. Четвериков, Симметрии и законы сохранения уравнений математической физики, Факториал, М., 2005.

[5] T. Voronov, "Graded manifolds and Drinfeld doubles for Lie bialgebroids", Quantization, Poisson Brackets and Beyond, Contemp. Math., 315, ed. T. Voronov, AMS, Providence, RI, 2002, 131-168.

[6] J.-C. Herz, C. R. Acad. Sci. Paris, 263 (1953), 1935-1937; 2289-2291.

[7] Y. Kosmann-Schwarzbach, F. Magri, Ann. Inst. H. Poincaré, 53:1 (1990), 35-81.

[8] А. В. Киселев, Й. В. ван де Лёр, ТМФ, 162:2 (2010), 179-195, arXiv: 0902.3624.

[9] A. N. Leznov, M. V. Saveliev, Lett. Math. Phys., 3:6 (1979), 489-494.

[10] А. В. Жибер, В. В. Соколов, УМН, 56:1(337) (2001), 63-106.

[11] A. Kumpera, D. C. Spencer, Lie Equations, v. I: General theory, Ann. Math. Stud., 73, Princeton Univ. Press, Princeton, N.J.; Univ. Tokyo Press, Tokyo, 1972.

[12] J. Krasil'shchik, A. Verbovetsky, "Geometry of jet spaces and integrable systems", J. Geom. Phys. (в печати), arXiv: 1002.0077.

[13] B. A. Kupershmidt, "Geometry of jet bundles and the structure of Lagrangian and Hamiltonian formalisms", Geometric Methods in Mathematical Physics, Lecture Notes in Math., 775, Springer, Berlin, 1980, 162-218. 
[14] П. Олвер, Приложения групп Ли к дифберенииальным уравнениям, Мир, М., 1989.

[15] А. В. Киселев, ТМФ, 144:1 (2005), 83-93, arXiv: nlin.SI/0409061.

[16] A. V. Kiselev, J. W. van de Leur, J. Phys. A, 42:40 (2009), 404011, 8 pp., arXiv: 0903.1214.

[17] A. V. Kiselev, J.W. van de Leur, Involutive distributions of operator-valued evolutionary vector fields and their affine geometry, Preprint IHES/M/07/38, Inst. Hautes Études Sci., Bures-sur-Yvette.

[18] P. Kersten, I. Krasil'shchik, A. Verbovetsky, J. Geom. Phys., 50:1-4 (2004), 273-302, arXiv: math/0304245.

[19] M. Nesterenko, R. Popovych, J. Math. Phys., 47:12 (2006), 123515, 45 pp., arXiv: math-ph/0608018.

[20] C. P. Boyer, J. D. Finley III, J. Math. Phys., 23:6 (1982), 1126-1130.

[21] В. А. Головко, И. С. Красильщик, А. М. Вербовецкий, ТМФ, 154:2 (2008), 268-282, arXiv: 0812.4684.

[22] T. Lada, J. Stasheff, Internat. J. Theoret. Phys., 32:7 (1993), 1087-1103, arXiv: hep-th/9209099.

[23] А.В. Киселев, Фундамент. и прикл. матем., 11:1 (2005), 159-180, arXiv: math.RA/0410185.

[24] P. Kersten, I. Krasil'shchik, A. Verbovetsky, Acta Appl. Math., 83:1-2 (2004), 167-173, arXiv: math/0310451.

[25] П. А. М. Дирак, Лекиии по квантовой механике, Мир, М., 1968; T. de Donder, Théorie invariantive du calcul des variations, Gauthier-Villars, Paris, 1930.

[26] И. С. Красильщик, А. М. Вербовецкий, Гомологические методъ в теории уравнений математической физики, 1999, arXiv: math.DG/9808130. 